\title{
Evaluation of Signal-to-Background and Mg II/Mg I Ratios as Response for the Optimization of Rare Earth Elements Determination by Inductively Coupled Plasma Optical Emission Spectrometry
}

\author{
Aline K. Guimarães-Silva, ${ }^{a, c}$ Jorge C. de Lena, ${ }^{a}$ Roberta E. S. Froes, ${ }^{b}$ \\ Letícia M. Costa and Clésia C. Nascentes*, \\ ${ }^{a}$ Departamento de Geologia and ${ }^{b}$ Departamento de Química,Universidade Federal de Ouro Preto, \\ Campus Morro do Cruzeiro, s/n, 35400-000 Ouro Preto-MG, Brazil \\ 'Departamento de Química, Instituto de Ciências Exatas, Universidade Federal de Minas Gerais, \\ Av. Antonio Carlos, 6627, 31270-901 Belo Horizonte-MG, Brazil
}

\begin{abstract}
Elementos terras raras (ETR) fazem parte de um grupo peculiar de elementos químicos e sua determinação em amostras geológicas é importante e complexa, devido às baixas concentrações e problemas com interferências espectrais e não espectrais. Neste trabalho, planejamentos experimentais foram utilizados para otimizar as condições operacionais de um espectrômetro de emissão óptica com plasma indutivamente acoplado (ICP OES) para a determinação de ETR. A razão entre o sinal analítico dos ETR e o sinal de fundo (SBR) e a razão $\mathrm{Mg}$ II/Mg I foram avaliadas como respostas na otimização. De acordo com os resultados obtidos, três diferentes condições de operação foram selecionadas e classificadas como robusta, semi-robusta e melhor SBR. Limites de detecção (LOD), SBR e desvio padrão relativo de medidas de uma solução do branco $\left(\mathrm{DPR}_{\text {branco }}\right)$ foram determinados. Na condição semi-robusta (potência de $1250 \mathrm{~W}$ e vazão do gás nebulizador de $0,90 \mathrm{~L} \mathrm{~min}^{-1}$ ) foram obtidos $\mathrm{LOD}$ e $\mathrm{DPR}_{\text {branco }}$ mais baixos que nas outras condições estabelecidas.
\end{abstract}

Rare earth elements (REEs) is a peculiar group of chemical elements. Their determination in geological samples is important and complex due to their low concentrations and problems with spectral and non-spectral interferences. Herein, the operating conditions of an inductively coupled plasma optical emission spectrometer were optimized for the determination of REEs using experimental designs. The signal-to-background ratio (SBR) of the REEs emission signals and the $\mathrm{Mg} \mathrm{II} / \mathrm{Mg}$ I line intensity ratio were evaluated as response functions. According to the results obtained, three different sets of operating conditions were selected and classified as robust, semi-robust and best SBR conditions. Limits of detection (LOD), SBR and relative standard deviation of the blank solution ( $\mathrm{RSD}_{\text {blank }}$ ) were determined. The semi-robust condition (RF power of $1250 \mathrm{~W}$ and nebulizer gas flow rate of $0.90 \mathrm{~L} \mathrm{~min}^{-1}$ ) exhibited lower LOD and $\mathrm{RSD}_{\text {blank }}$ than others tested operating conditions.

Keywords: rare earth elements, ICP OES, optimization, SBR, Mg II/Mg I

\section{Introduction}

Inductively coupled plasma optical emission spectrometry (ICP OES) has been widely used for multielement analysis and is recognized as one of the most effective techniques for determination of major, minor and trace elements including the rare earth elements (REEs) in geological samples with complex matrices. ${ }^{1-4}$ Recently,

*e-mail: clesia@qui.ufmg.br inductively coupled plasma mass spectrometry (ICP-MS) has shown to be a powerful tool for the direct determination of REEs, but it is still too expensive and unavailable for many laboratories. On the other hand, ICP OES is more affordable and shows good analytical qualities, such as precision and accuracy, relatively low limits of detection, high sample throughput, simultaneous determination of most elements and wide dynamic range..$^{5-7}$

From a geological point of view, the determination of REEs is vital to understand the geochemical processes in 
which rocks have been formed and to provide information about the environment. ${ }^{8-10}$ Several instrumental techniques are currently employed for REEs determination such as instrumental neutron activation analysis (INAA), isotopic dilution mass-spectrometry (ID-MS), atomic absorption spectrometry (AAS), X-ray fluorescence (XRF), inductively coupled plasma optical emission spectrometry (ICP OES) and inductively coupled plasma mass spectrometry (ICP-MS). Frequently the determination of almost all REEs is carried out by a combination of methods, including chemical separation..$^{11,12}$

Geological materials present some analytical challenges related to complex matrix composition and low REEs concentrations in most samples. The main constituents in a geological sample (rocks, soils and sediments) are $\mathrm{Si}, \mathrm{Al}, \mathrm{Fe}$, $\mathrm{Ti}, \mathrm{Mn}, \mathrm{Mg}, \mathrm{Ca}, \mathrm{Na}$ and $\mathrm{K}$. These elements can contribute to high levels of chemical or spectral interferences and spurious background emissions due to the matrix complexity. Aluminum, $\mathrm{Ca}, \mathrm{Fe}$ and to a lesser extesion $\mathrm{Ti}$ can cause significant background in many regions of the spectrum or give rise to serious line overlap interferences in the determination of trace elements. ${ }^{13}$ Easily ionized elements (EIEs), such as alkalis, $\mathrm{Na}$ and $\mathrm{K}$, with their low first ionization potential can cause a shift in the spatial distribution of analyte-ionization equilibrium. ${ }^{14-16}$ Calcium, $\mathrm{Ti}$ and $\mathrm{Mn}$ are elements with a low second ionization potential that can cause severe matrix effects. ${ }^{17,18}$ These effects on the REEs emission signal will be discussed in a future work. In order to overcome some of these restrictions, sample pretreatment procedures have been extensively employed to separate and preconcentrate analytes prior to analysis. ${ }^{19}$ The optimization of the operating conditions is also an important step in the analytical sequence to guarantee the best analytical performance. The goal of any analytical procedure is to achieve a measurement with high level of sensitivity, great accuracy and precision, and the lowest limit of detection. Furthermore, the measurement technique should be robust. ${ }^{20}$

Good sensitivity is required for the determination of REEs in geological samples because these trace elements are found in complex matrices. Also, robustness is an important factor in the optimization of the ICP since the plasma must be able to deal with a matrix change without affecting plasma conditions, such as temperature and electron number density. Plasma conditions are easily evaluated using $\mathrm{Mg}$ II $(280 \mathrm{~nm})$ and $\mathrm{Mg}$ I (285 nm) line intensity ratio, ${ }^{21,22}$ a parameter that has gained almost universal popularity in the ICP OES community. ${ }^{23}$ The signal-to-background ratio (SBR) is another parameter commonly applied since it can be correlated to limits of detection. ${ }^{24}$ Choosing one parameter instead of another could lead to the use of a different set of operating conditions.

ICP has a wide variety of possible optimization parameters, including the operating conditions and instrumental variations related to types of torches and sample introduction system. Some factors have an expressive influence on the plasma excitation conditions, such as radio frequency (RF) incident power, nebulizer gas flow rate, auxiliary gas flow rate, plasma gas flow rate, sample uptake rate and observation height. ${ }^{13,24}$ In ICP OES, when radial view is used, at least three parameters must be taken into account: incident applied power, nebulizer gas flow rate and observation height.

Most of the studies about the determination of REEs deals with the optimization of the separation and preconcentration step, but ICP OES operating conditions are not optimized. These operating condition are only cited, sometimes based on previous works, such as the paper of Walsh et al.. ${ }^{25}$ This is quite common in geochemical studies since the main purpose is the interpretation of the REEs pattern in environmental samples and not the development and the optimization of methodology. The optimization of ICP OES operating conditions for the determination of REEs has received little attention and there are not many works in the literature about the behavior of these elements in different conditions. Conversely, major and trace elements, specially toxic elements like $\mathrm{As}, \mathrm{Cd}, \mathrm{Cr}$, $\mathrm{Hg}$, and $\mathrm{Pb}$ are extensively discussed in the literature. ${ }^{26-30}$

In this work, the operating conditions of ICP OES were optimized aiming for reaching the best conditions for REEs determination ( $\mathrm{La}, \mathrm{Nd}, \mathrm{Eu}, \mathrm{Gd}, \mathrm{Dy}, \mathrm{Er}$ and $\mathrm{Yb}$ ) based on robustness and sensitivity. Two different parameters were evaluated and compared as responses to obtain the optimum conditions: $\mathrm{Mg} \mathrm{II/Mg}$ I line intensity ratio and SBR values of the REEs. Doehlert design was applied for the optimization of radio frequency applied power, nebulizer gas flow rate, sample uptake rate and observation height.

\section{Experimental}

Instrumentation

All measurements were performed with a simultaneous ICP OES spectrometer Spectro (Germany), model Ciros CCD with radial view. A Scott type double-pass spray chamber and a cross-flow nebulizer were used throughout the experiments. The detailed description of the equipment and operational parameters are presented in Table 1. The wavelengths selected and the ionization and excitation energies are reported in Table 2. 
Table 1. ICP OES instrumental operating conditions

\begin{tabular}{ll}
\hline Instrument & $\begin{array}{l}\text { Spectro Ciros CCD } \\
\text { Paschen-Runge mount } \\
\text { Polychromator }\end{array}$ \\
Optical grating/Line density & $\begin{array}{l}2924 \text { grooves } \mathrm{mm}^{-1}-160 \text { to } 460 \mathrm{~nm} \\
2400 \text { grooves } \mathrm{mm}^{-1}-588 \text { to } 766 \mathrm{~nm}\end{array}$ \\
& $160-770 \mathrm{~nm}$ \\
Wavelength range & $500 \mathrm{~mm}$ \\
Focal distance & Cross-flow with Scott-type spray \\
Nebulizer type & chamber \\
Instrumental Parameters & $1100-1400 \mathrm{~W}$ \\
RF applied power & $12.0 \mathrm{~L} \mathrm{~min}{ }^{-1}$ \\
Coolant gas flow rate & $1.0 \mathrm{~L} \mathrm{~min}{ }^{-1}$ \\
Auxiliar gas flow rate & $0.6-1.2 \mathrm{~L} \mathrm{~min}^{-1}$ \\
Nebulizer gas flow rate & $1-3 \mathrm{~mL} \mathrm{~min}$ m $^{-1}$ \\
Sample uptake rate & $13 \mathrm{~mm}$ \\
Observation heigth & $1.8 \mathrm{~mm}$ \\
Inner diameter of the torch & \\
injector & $21.5 \mathrm{~s} \mathrm{(manual)}$ \\
Data acquisition / time &
\end{tabular}

The selection includes 7 elements and 4 or 5 ionic lines for each element, and the sum of ionization and excitation energies of the lines ranging from 8.40 to $13.41 \mathrm{eV}$. Analytical lines were selected based on sensitivity and minimum spectral interference. Spectral line interferences due to $\mathrm{Si}, \mathrm{Al}, \mathrm{Ti}, \mathrm{Fe}, \mathrm{Mn}, \mathrm{Mg}, \mathrm{Ca}, \mathrm{Na}$ and $\mathrm{K}$ were evaluated by scanning the spectra of the analytes and the interfering elements using the profile mode of the analysis program. An improper analytical line selection may result in significant loss of detection power or accuracy for samples containing complex matrices. Therefore, the appropriate choice of analytical line is crucial to avoid interferences and ensure the quality of analysis. ${ }^{31}$

\section{Reagents, samples and test solutions}

High-purity water was supplied from a Millipore Milli-Q ion-exchange system (Millipore, Billerica, MA, USA). The concentrated nitric, hydrochloric and hydrofluoric acids employed were of analytical-reagent grade (Merck, Darmstadt, Germany). Laboratory glassware was kept overnight in a $10 \%(\mathrm{v} / \mathrm{v})$ nitric acid solution and before use the glassware was rinsed with ultrapure water. Reference solutions were prepared from $1 \mathrm{~g} \mathrm{~L}^{-1}$ certified standard solutions (J. T. Baker, Phillipsburg, NJ, USA) for REEs ( $\mathrm{La}, \mathrm{Nd}, \mathrm{Eu}, \mathrm{Gd}$, Dy, Er and $\mathrm{Yb}$ ) and for major common constituents in geological matrices ( $\mathrm{Al}, \mathrm{Fe}, \mathrm{Mn}$, $\mathrm{Ti}, \mathrm{Na}, \mathrm{K}, \mathrm{Ca}$ and $\mathrm{Mg}$ ).

The emission signals were evaluated in solutions with and without the matrix medium. The concentrations of the concomitants in test solutions were prepared according to typical mean concentrations presented in relevant geological
Table 2. Line selection, ionization energy $\left(\mathrm{E}_{\mathrm{ion}}\right)$, excitation energy $\left(\mathrm{E}_{\text {exc }}\right)$, and the sum of the ionization and excitation energies $\left(\mathrm{E}_{\text {sum }}\right)$, for rare earth elements. ${ }^{32}$ Energy is expressed in $\mathrm{eV}$.

\begin{tabular}{lcccc}
\hline Line / nm & $\mathrm{E}_{\text {exc }}$ & $\mathrm{E}_{\text {ion }}$ & $\mathrm{E}_{\text {sum }}$ & $\begin{array}{c}\text { Relative } \\
\text { Intensity }\end{array}$ \\
\hline La II 333.749 & 4.12 & 5.58 & 9.70 & 1500 \\
La II 379.478 & 3.51 & 5.58 & 9.09 & 3900 \\
La II 408.672 & 3.03 & 5.58 & 8.61 & 5500 \\
La II 412.323 & 3.33 & 5.58 & 8.91 & 4400 \\
La II 419.655 & 3.27 & 5.58 & 8.85 & 1500 \\
Nd II 401.225 & 3.72 & 5.52 & 9.24 & 3700 \\
Nd II 406.109 & 3.52 & 5.52 & 9.04 & 4700 \\
Nd II 417.732 & 3.03 & 5.52 & 8.55 & 2400 \\
Nd II 430.358 & 2.88 & 5.52 & 8.40 & 5400 \\
Eu II 372.494 & 3.33 & 5.67 & 9.00 & $20000^{\mathrm{a}}$ \\
Eu II 390.710 & 3.38 & 5.67 & 9.05 & $28000^{\mathrm{a}}$ \\
Eu II 393.048 & 3.36 & 5.67 & 9.03 & $32000^{\mathrm{a}}$ \\
Eu II 412.970 & 3.00 & 5.67 & 8.67 & $33000^{\mathrm{a}}$ \\
Eu II 420.505 & 2.95 & 5.67 & 8.62 & $60000^{\mathrm{a}}$ \\
Gd II 335.047 & 3.84 & 6.15 & 9.99 & 5400 \\
Gd II 335.862 & 3.72 & 6.15 & 9.87 & 4300 \\
Gd II 336.223 & 3.76 & 6.15 & 9.91 & 5400 \\
Gd II 342.247 & 3.86 & 6.15 & 10.01 & 6900 \\
Dy II 340.780 & 3.64 & 5.94 & 9.58 & 5300 \\
Dy II 353.170 & 3.51 & 5.94 & 9.45 & 22000 \\
Dy II 353.602 & 4.04 & 5.94 & 9.98 & 5500 \\
Dy II 394.468 & 3.14 & 5.94 & 9.08 & 10000 \\
Dy II 400.045 & 3.20 & 5.94 & 9.14 & 8000 \\
Er II 323.058 & 3.89 & 6.11 & 10.00 & 2300 \\
Er II 326.478 & 3.80 & 6.11 & 9.91 & 2700 \\
Er II 349.910 & 3.60 & 6.11 & 9.71 & 6700 \\
Er II 369.265 & 3.41 & 6.11 & 9.52 & 7900 \\
Er II 390.631 & 3.17 & 6.11 & 9.28 & 11000 \\
Yb II 275.048 & 7.16 & 6.25 & 13.41 & 1300 \\
Yb II 297.056 & 4.17 & 6.25 & 10.42 & 2000 \\
Yb II 328.937 & 3.77 & 6.25 & 10.02 & 18000 \\
Yb II 369.419 & 3.35 & 6.25 & 9.60 & 32000 \\
\hline CO $3510 x a n d$ & & & \\
\hline
\end{tabular}

aComplex and wide line.

samples routinely analyzed in the laboratory. Final solutions were acidified with $5 \%(\mathrm{v} / \mathrm{v}) \mathrm{HNO}_{3}$. Digests from geological samples was mixed to use in some spectra measurements, considering the effect of the matrix medium in the emission signal of REEs. Two certified reference materials (CRMs) were used to evaluate the accuracy in the proposed operating conditions: AC-E (granite) from Centre de Recherches Pétrographiques et Géochimiques - CNRS (Vandoeuvre-lèsNancy, France) and GBW 07309 (stream sediment) from the National Research Centre (Hepingjie, China).

\section{Sample preparation}

The sample preparation procedure for geological samples was previously described by Moutte. ${ }^{33}$ A $250 \mathrm{mg}$ portion of sample was directly and accurately weighted into 
a $30 \mathrm{~mL}$ Savillex molded perfluoralkoxy (PFA) vessels. The decomposition was carried out in hot plate device and was developed with successive additions of $\mathrm{HCl}\left(10 \mathrm{~mol} \mathrm{~L}^{-1}\right)$, $\mathrm{HNO}_{3}\left(10 \mathrm{~mol} \mathrm{~L}^{-1}\right)$ and concentrated HF. Then, the residue was dissolved to $25 \mathrm{~mL}$ using $2 \mathrm{~mol} \mathrm{~L}^{-1} \mathrm{HCl}$ solution.

\section{Optimization procedure}

Doehlert experimental design was used to optimize the operating parameters. The RF applied power, nebulizer gas flow rate, sample uptake rate and observation height were selected as independent variables. The $\mathrm{Mg} \mathrm{II} / \mathrm{Mg}$ I line intensity ratio and SBR values for REEs were considered as responses. The SBR value is given by SBR = (total signal background)/background.The background was measured from a separate measurement of the blank (i.e., on-peak background). Table 3 shows the design proposed and the respective parameters values. According to this design, each variable was studied at least in three levels. The central point was made in five replicates, adding up to 25 experiments. The maximum and minimum levels of each factor were established taking into account the default operating conditions and data of previous univariate experiments.

The experimental data was processed using STATISTICA ${ }^{\circledR}$ software version 6.0 (StatSoft, Tulsa, OK, USA). The aim of the experiments carried out was not to define mathematical models, but to verify the influence of the operational variables on the emission signals. All measurements were carried out using a test solution with $1 \mathrm{mg} \mathrm{L}^{-1}$ of each REEs and $5 \mathrm{mg} \mathrm{L}^{-1} \mathrm{Mg}$. In order to check matrix effects on robustness and sensitivity, a multielement solution containing $\mathrm{Al} 800 \mathrm{mg} \mathrm{L}^{-1}$, Fe $600 \mathrm{mg} \mathrm{L}^{-1}$, Mn $25 \mathrm{mg} \mathrm{L}^{-1}$, Ti $80 \mathrm{mg} \mathrm{L}^{-1}$, Na $200 \mathrm{mg} \mathrm{L}^{-1}$, K $200 \mathrm{mg} \mathrm{L}^{-1}$, $\mathrm{Ca} 400 \mathrm{mg} \mathrm{L}^{-1}$ and $\mathrm{Mg} 400 \mathrm{mg} \mathrm{L}^{-1}$, was employed using the same design to simulate the matrix medium. Magnesium is a test element for the evaluation of robustness, consequently, in the matrix medium, the $\mathrm{Mg}$ concentration in the solution was only $5 \mathrm{mg} \mathrm{L}^{-1}$.

Table 3. Design matrix for Doehlert model: experimental variables and levels for ICP OES procedure

\begin{tabular}{|c|c|c|c|c|c|c|c|c|}
\hline \multicolumn{4}{|c|}{ Variable } & Levels & $(-)$ & $\mathrm{CP}$ & $(+)$ & Unit \\
\hline 1 & \multicolumn{3}{|c|}{ Observation heigth } & 5 & 9 & 11.5 & 14 & $\mathrm{~mm}$ \\
\hline 2 & \multicolumn{3}{|c|}{ RF applied power } & 7 & 1100 & 1250 & 1400 & $\mathrm{~W}$ \\
\hline 3 & \multicolumn{3}{|c|}{ Nebulizer gas flow rate } & 7 & 0.6 & 0.9 & 1.2 & $\mathrm{~L} \min ^{-1}$ \\
\hline 4 & \multicolumn{3}{|c|}{ Sample uptake rate } & 3 & 1 & 2 & 3 & $\mathrm{~mL} \min ^{-1}$ \\
\hline \multirow{2}{*}{ Experiment } & \multicolumn{4}{|c|}{ Code Value } & \multicolumn{4}{|c|}{ Real Value } \\
\hline & 1 & 2 & 3 & 4 & 1 & 2 & 3 & 4 \\
\hline$\overline{1}$ & 0 & 0 & 0 & 0 & 11.5 & 1250 & 0.90 & 2 \\
\hline 2 & 1 & 0 & 0 & 0 & 9.0 & 1250 & 0.90 & 2 \\
\hline 3 & 0.5 & 0.866 & 0 & 0 & 15.8 & 1400 & 0.90 & 2 \\
\hline 4 & 0.5 & 0.289 & 0.817 & 0 & 15.8 & 1300 & 1.20 & 2 \\
\hline 5 & 0.5 & 0.289 & 0.204 & 0.791 & 15.8 & 1300 & 0.97 & 3 \\
\hline 6 & -1 & 0 & 0 & 0 & 14 & 1250 & 0.90 & 2 \\
\hline 7 & -0.5 & -0.866 & 0 & 0 & 13.3 & 1100 & 0.90 & 2 \\
\hline 8 & -0.5 & -0.289 & -0.817 & 0 & 13.3 & 1200 & 0.60 & 2 \\
\hline 9 & -0.5 & -0.289 & -0.204 & -0.791 & 13.3 & 1200 & 0.82 & 1 \\
\hline 10 & 0.5 & -0.866 & 0 & 0 & 15.8 & 1100 & 0.90 & 2 \\
\hline 11 & 0.5 & -0.289 & -0.817 & 0 & 15.8 & 1200 & 0.60 & 2 \\
\hline 12 & 0.5 & -0.289 & -0.204 & -0.791 & 15.8 & 1200 & 0.82 & 1 \\
\hline 13 & -0.5 & 0.866 & 0 & 0 & 13.3 & 1400 & 0.90 & 2 \\
\hline 14 & 0 & 0.577 & -0.817 & 0 & 11.5 & 1350 & 0.60 & 2 \\
\hline 15 & 0 & 0.577 & -0.204 & -0.791 & 11.5 & 1350 & 0.82 & 1 \\
\hline 16 & -0.5 & 0.289 & 0.817 & 0 & 13.3 & 1300 & 1.20 & 2 \\
\hline 17 & 0 & -0.577 & 0.817 & 0 & 11.5 & 1150 & 1.20 & 2 \\
\hline 18 & 0 & 0 & 0.613 & -0.791 & 11.5 & 1250 & 1.12 & 1 \\
\hline 19 & -0.5 & 0.289 & 0.204 & 0.791 & 13.3 & 1300 & 0.97 & 3 \\
\hline 20 & 0 & -0.577 & 0.204 & 0.791 & 11.5 & 1150 & 0.97 & 3 \\
\hline 21 & 0 & 0 & -0.613 & 0.791 & 11.5 & 1250 & 0.67 & 3 \\
\hline 22 & 0 & 0 & 0 & 0 & 11.5 & 1250 & 0.90 & 2 \\
\hline 23 & 0 & 0 & 0 & 0 & 11.5 & 1250 & 0.90 & 2 \\
\hline 24 & 0 & 0 & 0 & 0 & 11.5 & 1250 & 0.90 & 2 \\
\hline 25 & 0 & 0 & 0 & 0 & 11.5 & 1250 & 0.90 & 2 \\
\hline
\end{tabular}




\section{Results and Discussion}

Robustness - Mg II/Mg I line intensity ratio

The Doehlert design was used to evaluate robustness. The use of multivariate techniques for the optimization of operational conditions in spectroscopic methods has been thoroughly exploited by analytical chemists, as evidenced by the multiple advantages that can be found using these tools. $^{34,35}$ The operating parameters were considered as factors: RF incident applied power, nebulizer gas flow rate, sample uptake rate and observation height. The response surface in Figure 1a illustrates the influence of main factors on the response ( $\mathrm{Mg} \mathrm{II} / \mathrm{Mg}$ I line intensity ratio) when a solution containing only $\mathrm{Mg}$ and REEs was analyzed. The nebulizer gas flow rate and RF applied power were found to be the most significant factors. A robust condition was obtained at high RF power and low nebulizer gas flow rate, as expected and described in the literature. ${ }^{36-38} \mathrm{~A}$ response surface maximum point was not obtained; an ever-increasing pattern was observed until the instrumental limits. The thresholds between robust and non-robust conditions were established.

Also, the robustness was tested in a simulated multielement solution with the common constituents of a geological matrix in the respective concentrations (in mg L ${ }^{-1}$ ): Al 800, Fe 600, Ti 80, Mn 25, Na 200, K 200, and $\mathrm{Ca} 400$. These concentrations are commonly observed in samples analyzed in routine studies of the laboratory. Magnesium was the test element and was considered at the concentration of $5 \mathrm{mg} \mathrm{L}^{-1}$. It was possible to observe a decrease in the value of $\mathrm{Mg} \mathrm{II} / \mathrm{Mg} \mathrm{I}$, but the general trend is the same determined to the solution without matrix (Figures 1a and 1b).

The main factors had the same influence in the robustness, and a maximum point was not obtained. There was a mean decrease in $\mathrm{Mg}$ II/Mg I line intensity ratio of about $8 \%$. Therefore, to maintain robust conditions in the presence of the matrix medium, higher RF applied power and lower nebulizer gas flow rate should be considered. If the optimization process was carried out with an aqueous solution without matrix medium, then the $\mathrm{Mg}$ II/Mg I intensity ratio above 9 should be needed to guarantee robust conditions in matrix medium. Both designs demonstrated that above $90 \%$ of the variance can be explained by the proposed model in the evaluation of robustness.

Mermet and co-workers ${ }^{22,39}$ defined robustness as the capability of the ICP system to accept a change in the concentration of major elements, acids, and other elements, without any significant variation in the line intensity of the analytes. This concept has become quite common in ICP (a) Without Matrix Solution

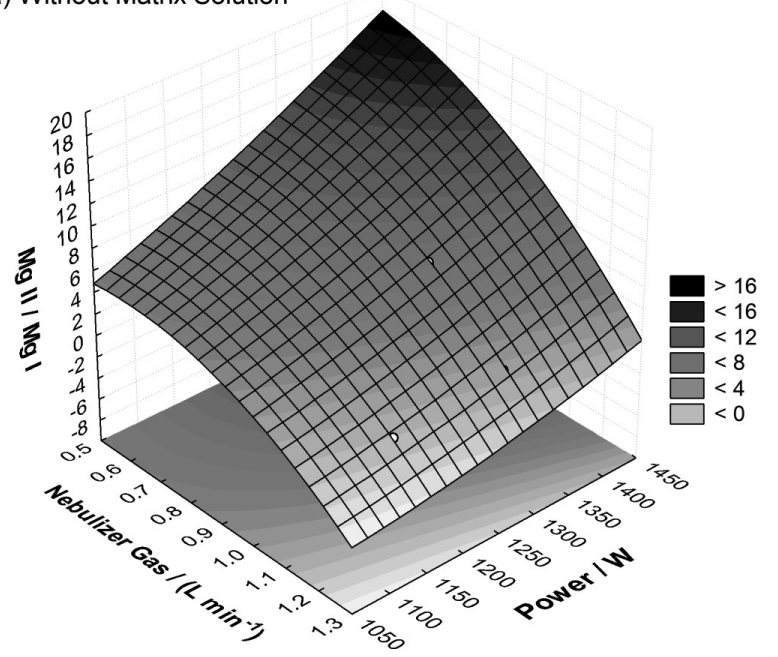

(b) With Matrix Solution

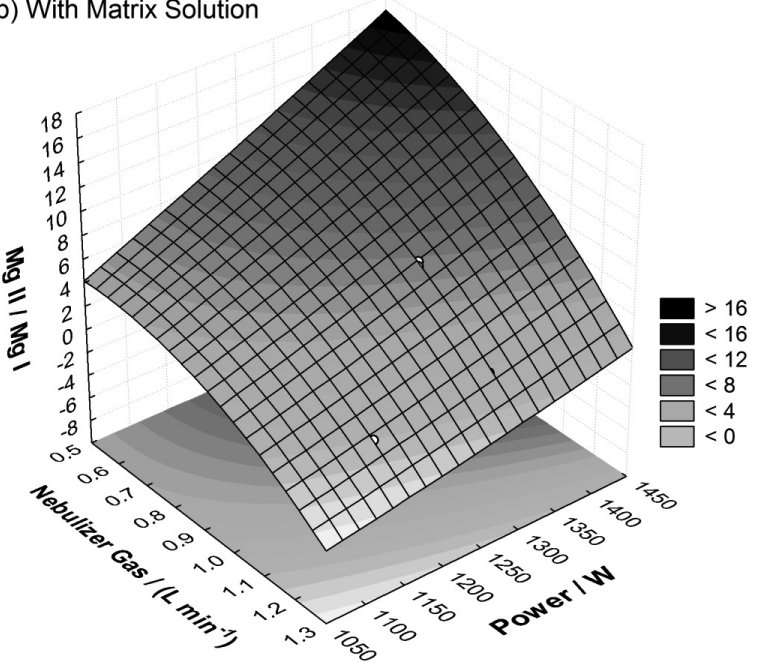

Figure 1. Effect of RF applied power and nebulizer gas flow rate on $\mathrm{Mg} \mathrm{II} / \mathrm{Mg}$ I line intensity ratio (a) without and (b) with the matrix solution.

optimization studies and can be easily measured using ionic-to-atomic line intensity ratio as $\mathrm{Mg}$ II (280 nm) and Mg I (285 nm). ${ }^{27,36,40-42}$ Since ionic lines are more sensitive to changes in atomization and excitation conditions than atomic lines, the intensity ratio of the lines is a practical criterion to evaluate the plasma robustness and analytical performance. A robust condition can be achieved when higher RF applied power level ( $\geq 1200 \mathrm{~W}$ ) is combined with lower nebulizer gas flow rate $\left(\leq 0.6 \mathrm{~L} \mathrm{~min}^{-1}\right)^{37,38,43,44}$ and this condition is characterized by the high intensity ratio of $\mathrm{Mg} \mathrm{II} / \mathrm{Mg} \mathrm{I}$, values above $8 .^{22}$

\section{Sensitivity -signal-to-background ratio (SBR)}

Because of the spectral complexity, low sensitivity and interference combined with the low abundance of REEs, a set of operating condition should be considered 
to improve the signal-to-background ratio during the determination of REEs in geological samples. The Doehlert design demonstrated that the main factors which are able to influence the SBR value of the REEs were RF applied power and nebulizer gas flow rate as well as for robustness. However, in this case, the RF incident power was more significant than the nebulizer gas flow rate. The RF applied power is directly linked to the amount of energy transferred to the species and has a direct influence on net signal intensities and SBR value. ${ }^{45}$ Unlike what was observed for robustness, both parameters demonstrated negative estimated effects leading to a set of operating conditions with lower RF applied power and intermediate nebulizer gas flow rate to obtain higher SBR values.

Response surfaces were obtained for each element by plotting the SBR of each element against the RF applied power and nebulizer gas flow rate. The results were obtained for solutions containing REEs in presence and absence of matrix. It was possible to observe the uniform behavior of SBR values in relation to the variation of the operating conditions. All analytical lines considered for REEs exhibited a similar distribution profile and the matrix tested did not significantly affect the value of SBR. Therefore a La line (408.672 nm) was chosen to exemplify the response surface obtained without and with matrix medium (Figures $2 \mathrm{a}$ and $2 \mathrm{~b}$, respectively). A maximum point was found at the operating conditions: $\mathrm{RF}$ applied power of $1100 \mathrm{~W}$ and nebulizer gas flow rate of $0.9 \mathrm{~L} \mathrm{~min}^{-1}$. Walsh et al..$^{25}$ also reported the application of a condition with lower-power to improve the SBR, which is a different condition commonly adopted to analyze major and trace elements in geological samples. Some papers published later cited this work as a reference condition to REEs. ${ }^{46}$ Others only cited the operating conditions adopted; in most cases, with RF applied power between $1000-1200$ W. $^{2,47}$ Recently, Bentlin and Pozebon ${ }^{48}$ reported the optimization related to the sample introduction system applying pneumatic and ultrasonic nebulization for the determination of REEs. In this work, the RF applied power and nebulizer gas flow rate were optimized only based on the Mg II/Mg I. Thus, a condition with higher RF applied power was applied considering values between 1300 and $1400 \mathrm{~W}$, and nebulizer gas flow rate was $0.75 \mathrm{~L} \mathrm{~min}^{-1}$.

In ICP OES, the experimental spectral intensity is a gross signal $(\mathrm{S}+\mathrm{B})$, which consists of the sum of the line intensity, $\mathrm{S}$, plus the background, $\mathrm{B}$. The background corresponds to the emission originating from the blank solution. Then the net signal is obtained by subtracting the value of the background, B. The simplest net signal (a) Without matrix solution

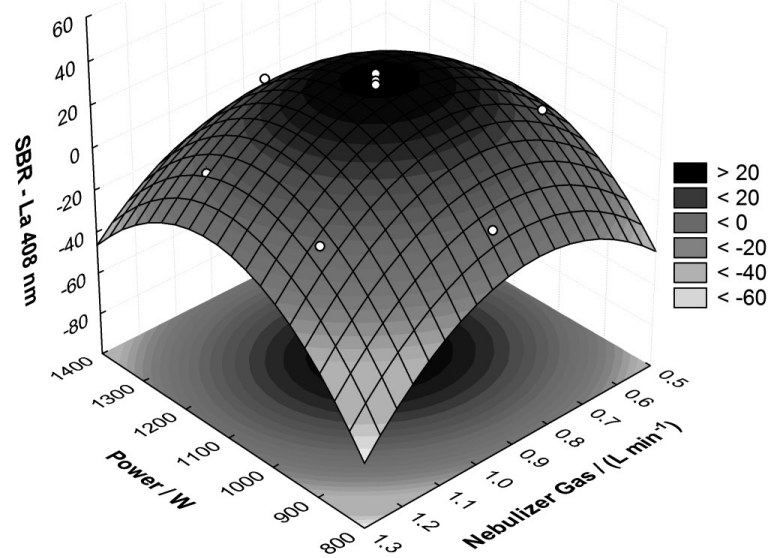

(b) With matrix solution

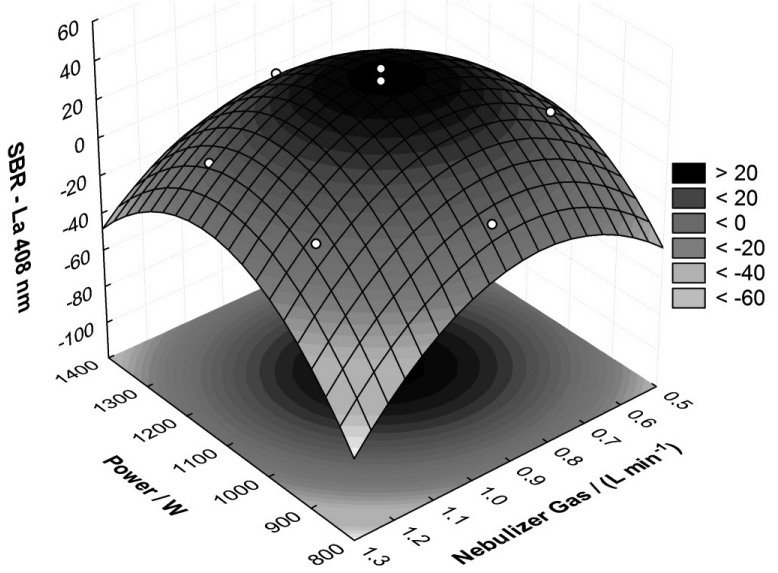

Figure 2. Effect of RF applied power and nebulizer gas flow rate on SBR value of La $408.672 \mathrm{~nm}$ in solution (a) without and (b) with matrix medium.

measurement consists of a direct measurement of the gross line intensity $\left(\mathrm{S}+\mathrm{B}_{\text {peak }}\right)$ at the central wavelength of the analyte line ${ }^{49}$ The signal will be processed unambiguously if the analytical signal is stand out from the background, which requires a meaning SBR value. The $\mathrm{SBR}$ is a traditional response used in optimization approach and it is also a figure of merit that can be correlated with limit of detection. ${ }^{24}$

It is important to consider the behavior of the background signal during the evaluation of SBR. There was an increase in analytical signals of REEs when increasing $\mathrm{RF}$ applied power, increasing nebulizer gas flow rate and at higher observation height. However, the background signal also had a sharp rise which led to a decrease in SBR values of the REEs (Figure 3a). Since an increase in power increases the background signals more than the analyte emission signals with subsequent decrease in SBR, it is assumed that a lower applied power seems to be the best choice for analyzing REEs by ICP OES. 
Instrumental operating conditions - robustness and sensitivity

When the operating conditions are established considering the sensitivity of the system based on SBR values, conditions with lower RF applied power settings would be applied and the robustness would be affected. In order to avoid this type of mistake, robustness and SBR ratio can be considered together during the optimization processes. In this case, robustness was defined by line intensity ratio of $\mathrm{Mg}$; on the other hand, SBR values were directly related to analytes. The Mg II/Mg I ratio and SBR values led to different sets of operating conditions because the emission lines considered in each response reacted differently to changes on excitation conditions. Evaluating the operating condition based on $\mathrm{Mg} \mathrm{II} / \mathrm{Mg}$ I the nebulizer gas flow rate was a more important parameter than the RF applied power. Conversely, considering the optimization with the SBR values of REEs the RF applied power was more significant and critical influence in the background was observed.

The main difference was related to changes in the RF applied power as can be seen in the behavior of the ionic lines of $\mathrm{Mg}$ and La in Figures $3 \mathrm{a}$ and $3 \mathrm{~b}$. Comparing REEs (e.g. La II $408 \mathrm{~nm}$ ) with magnesium (Mg II $280 \mathrm{~nm}$ ), an increase in RF applied power was followed by decreasing in SBR values of REEs and an increase in SBR value of $\mathrm{Mg}$. All REEs ionic lines studied revealed the same pattern. The behavior of analytical signal and SBR value can be affected by different factors as range of wavelength, ionization state, excitation energy for atomic lines and energy sum for ionic lines. In addition, the background emission signal had an important effect on the SBR. ${ }^{24,45}$ For some lines, the contribution of the background was higher than for others, probably because of the very low sensitivity of some lines. Non-robust conditions should be avoided for analytical applications dealing with complex matrices to guarantee precision and accuracy. But it cannot be the only one criterion in method development, mainly because it does not consider the behavior of the background emission signal that can be crucial in complex matrices.

The results obtained allowed the selection of three different sets of operating conditions (Table 4), classified on their robustness and the best SBR obtained. The routine operating condition is robust and is applied to determination of major and trace elements in environmental samples with good results. The best SBR condition was obtained when a lower RF applied power of $1100 \mathrm{~W}$ was applied. An intermediate condition between these two sets of operating conditions was suggested to consider robustness and sensitivity simultaneously. When a RF power of $1250 \mathrm{~W}$ was applied it is possible to obtain a semi-robust condition and intermediate values for SBR.

In ICP OES a measurement is based on the emission line intensity and this signal is composed of the net signal and the background signal. These signals exhibit different behaviors in face of changes in excitation and
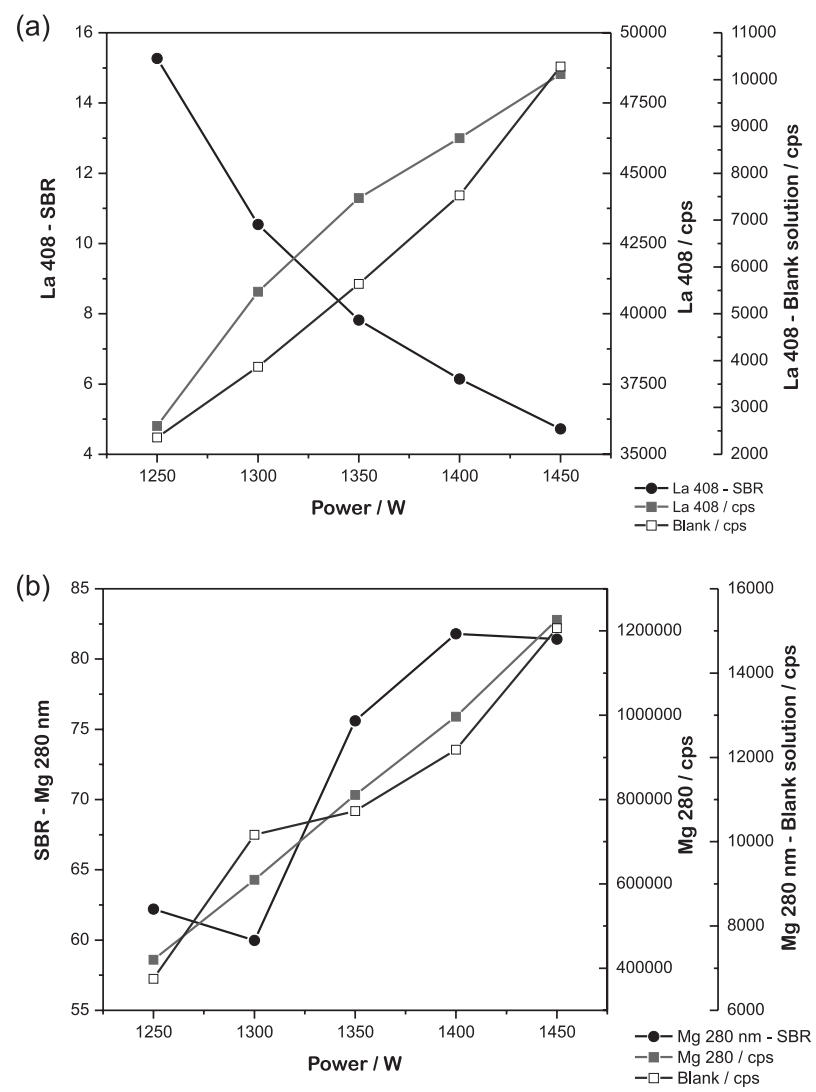

Figure 3. Analytical emission signal of (a) La II - $408 \mathrm{~nm}$ and (b) $\mathrm{Mg}$ II $-280 \mathrm{~nm}$ due to variation of RF applied power, at $0.9 \mathrm{~L} \mathrm{~min}^{-1}$

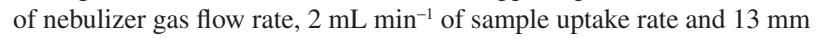
observation height.

Table 4. Identification of different set of operating conditions

\begin{tabular}{lccc}
\hline Parameter & \multicolumn{2}{c}{ Operating Conditions } \\
\cline { 2 - 4 } & (1) SBR 1100 W & (2) SBR 1250 W & (3) Robust 1350 W \\
\hline RF applied power / & 1100 & 1250 & 1350 \\
Nebulization gas flow rate / $\left(\mathrm{L} \mathrm{min}^{-1}\right)$ & 0.90 & 0.90 & 0.90 \\
Observation heigth / mm & 14 & 14 & 13 \\
Sample uptake rate / $\left(\mathrm{mL}\right.$ min $\left.^{-1}\right)$ & 2 & 2 & 2 \\
Mg II/Mg I ratio & 3 & 7 & 10 \\
\hline
\end{tabular}


atomization conditions and it should be considered during the optimization step. The behavior of the analytical signals (gross signal and background) for the REEs using the different sets of operating conditions were analyzed applying the emission line of La II 408.672 nm (Figure 4).

The samples analyzed consisted of a reference solution containing $1 \mathrm{mg} \mathrm{L}^{-1}$ of each REE, a blank solution and a mixture of digests from geological samples spiked with $1 \mathrm{mg} \mathrm{L}^{-1}$ of each REE. The pattern found for La II $(408.672 \mathrm{~nm})$ was also observed in others REEs emission lines. Considering the variation in the signal by reducing the RF applied power (1350-1100 W), it was also observed that the background signal suffers more influence when decreasing RF applied power. The analytical signal undergoes more change in Set $1(1100 \mathrm{~W})$, in fact it is a non-robust condition. Analyzing the matrix spectra (digest from geological samples spiked with REEs) the effect of decreasing the RF power was even more critical and the matrix effects were more significant.

Set 3 was considered a robust condition with lower SBR values and a considerable higher background signal (ca. 8000 cps, Figure 4 - Set 3). The results of the intermediate condition (Set 2) were clear in the spectrum; they displayed good sensitivity, low background emission and equivalent signals for reference and matrix solutions.

Table 5 shows the behavior of the 3 sets of operating conditions based on the values of limit of detection (LOD), SBR, relative standard deviation for 10 measurements of the blank solution ( $\mathrm{RSD}_{\text {blank }}$ ), and recoveries (\%) of REEs in reference solution (RS) and two certified reference materials, which were measured for the prominent lines considered in this work. The LOD was calculated using the background equivalent concentration (BEC) approach described by Thomsen et al..$^{50}$ The lowest background intensity was obtained in Set 1 (Figure 4), being $\mathrm{ca}$. 10-fold and 4-fold lower than Sets 3 and 2, respectively, and hence better SBR values. However, this condition shows a higher $\mathrm{RSD}_{\text {blank }}$ and lower $\mathrm{Mg}$ II/Mg I ratio - non-robust condition (Table 4), thus this condition was not used. The robust condition $(1350 \mathrm{~W})$ shows the highest LOD, the $\mathrm{RSD}_{\text {blank }}$ is higher than the Set 2 and the SBR value drops by half. The limiting factor in Set 3 - robust condition - is the highest LOD that could difficult the analysis of some REEs due to their low relative abundances. Besides that the quantitative results obtained for two certified reference materials does not show significant improvements in recoveries when comparing Set 2 and Set 3. Some interferences problems were not overcome regardless of the robust or better SBR condition, for example Gd and Er. Consequently, alternative approaches need to be investigated (standard additions method, internal standardization, mathematical
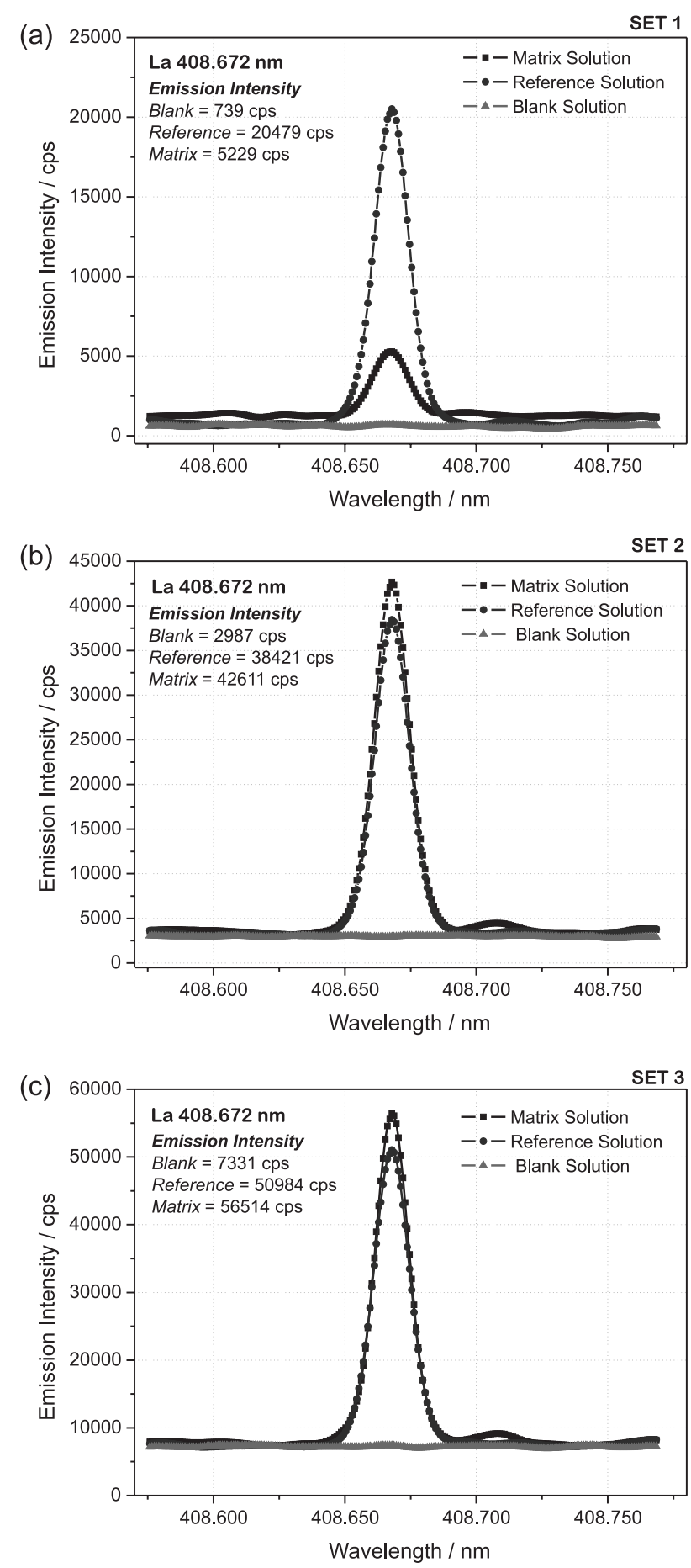

Figure 4. Spectral scan of La $408.672 \mathrm{~nm}$ at different sets of operating conditions. Set 1: $1100 \mathrm{~W}$; Set 2: $1250 \mathrm{~W}$; Set 3: $1350 \mathrm{~W}$.

corrections, etc.). So a detailed study of interferences in terms of major constituents of geological samples is ongoing and results will be presented later. Thus considering LOD, background signal and the need for interference correction regardless of the condition (robust or better SBR), it was clear that an intermediate condition between the robustness ( $\mathrm{Mg} \mathrm{II} / \mathrm{Mg}$ I) and sensitivity (SBR) would be a good choice 
Table 5. Limits of detection, SBR, relative standard deviation (RSD) of the blank solution for some REEs lines (ICP OES, Spectro Ciros CCD radially viewed) and recoveries (\%) of REEs in reference solution (RS) and two certified reference materials

\begin{tabular}{|c|c|c|c|c|c|c|c|}
\hline \multirow{2}{*}{\multicolumn{2}{|c|}{ Line and set of operating condition }} & \multirow{3}{*}{$\begin{array}{c}\text { LODs } /\left(\mu \mathrm{g} \mathrm{L}^{-1}\right) \\
5.9\end{array}$} & \multirow{3}{*}{$\begin{array}{l}\text { SBR } \\
6.42\end{array}$} & \multirow{3}{*}{$\begin{array}{c}\mathrm{RSD}_{\text {blank }} \\
1.27\end{array}$} & \multicolumn{3}{|c|}{ Recovery / \% } \\
\hline & & & & & \multirow{2}{*}{$\frac{\mathrm{RS}}{100.6}$} & \multirow{2}{*}{$\frac{\mathrm{AC}-\mathrm{E}}{103.1}$} & \multirow{2}{*}{$\begin{array}{c}\text { GBW07309 } \\
104.0\end{array}$} \\
\hline $\mathrm{La} 408 \mathrm{~nm}$ & $1350 \mathrm{~W}$ & & & & & & \\
\hline & $1250 \mathrm{~W}$ & 1.4 & 12.89 & 0.62 & 100.8 & 105.8 & 98.63 \\
\hline & $1100 \mathrm{~W}$ & 2.8 & 42.76 & 3.99 & 99.62 & 112.4 & 106.4 \\
\hline \multirow[t]{3}{*}{$\mathrm{Nd} 406 \mathrm{~nm}$} & $1350 \mathrm{~W}$ & 22.2 & 1.65 & 1.22 & 104.4 & 162.2 & 101.2 \\
\hline & $1250 \mathrm{~W}$ & 6.7 & 3.49 & 0.78 & 105.0 & 105.5 & 108.2 \\
\hline & $1100 \mathrm{~W}$ & 7.6 & 12.10 & 3.09 & 103.2 & 105.8 & 111.2 \\
\hline \multirow[t]{3}{*}{$\mathrm{Eu} 412 \mathrm{~nm}$} & $1350 \mathrm{~W}$ & 2.1 & 18.57 & 1.28 & 108.5 & 105.3 & 101.3 \\
\hline & $1250 \mathrm{~W}$ & 0.6 & 37.70 & 0.72 & 108.0 & 102.9 & 103.8 \\
\hline & $1100 \mathrm{~W}$ & 0.7 & 107.6 & 2.41 & 107.4 & 109.7 & 113.0 \\
\hline \multirow[t]{3}{*}{ Gd $342 \mathrm{~nm}$} & $1350 \mathrm{~W}$ & 5.7 & 4.68 & 0.90 & 102.0 & 117.7 & 294.2 \\
\hline & $1250 \mathrm{~W}$ & 2.4 & 8.32 & 0.68 & 103.0 & 125.6 & 297.1 \\
\hline & $1100 \mathrm{~W}$ & 4.2 & 16.43 & 2.28 & 100.3 & 135.2 & 311.2 \\
\hline \multirow[t]{3}{*}{ Dy $353 \mathrm{~nm}$} & $1350 \mathrm{~W}$ & 2.9 & 8.95 & 0.86 & 97.40 & 102.3 & 96.82 \\
\hline & $1250 \mathrm{~W}$ & 0.9 & 19.87 & 0.60 & 95.40 & 104.2 & 99.02 \\
\hline & $1100 \mathrm{~W}$ & 0.8 & 72.29 & 1.83 & 97.00 & 100.4 & 123.1 \\
\hline \multirow[t]{3}{*}{ Er 369 nm } & $1350 \mathrm{~W}$ & 7.5 & 4.62 & 1.15 & 108.0 & 97.46 & 52.82 \\
\hline & $1250 \mathrm{~W}$ & 1.3 & 9.67 & 0.43 & 104.8 & 94.80 & 73.54 \\
\hline & $1100 \mathrm{~W}$ & 1.6 & 29.46 & 1.58 & 107.6 & 95.31 & 85.35 \\
\hline \multirow[t]{3}{*}{ Yb $328 \mathrm{~nm}$} & $1350 \mathrm{~W}$ & 0.6 & 57.93 & 1.10 & 99.84 & 92.54 & 87.75 \\
\hline & $1250 \mathrm{~W}$ & 0.3 & 107.6 & 1.05 & 100.9 & 95.23 & 81.36 \\
\hline & $1100 \mathrm{~W}$ & 0.8 & 287.3 & 7.27 & 101.0 & 98.16 & 92.04 \\
\hline
\end{tabular}

RS: reference solution ( $\mathrm{La}, \mathrm{Nd} 500 \mu \mathrm{g} \mathrm{L} \mathrm{L}^{-1}$ and $\mathrm{Eu}, \mathrm{Gd}, \mathrm{Dy}, \mathrm{Er}, \mathrm{Yb} 250 \mu \mathrm{g} \mathrm{L}^{-1}$ ).

for analysis affording good LOD and SBR values, a low $\mathrm{RSD}_{\text {blank }}$ value and suitable recoveries.

\section{Conclusions}

This study focused on the optimization of the ICP OES aiming for better emission signals of REEs. The optimization process was based on two different responses: $\mathrm{Mg}$ II/Mg I line intensity ratio and SBR values, considering robustness and sensitivity. Robustness is a quite common response function in ICP OES optimization studies. Using different response functions for the optimization process of the operating conditions, such as robustness and sensitivity, the optimum conditions are not necessarily the same. An analytical judgment may be necessary to select the best operating conditions for complex samples. In the case of the REEs, it is very important to recognize their behavior in different conditions. Three different sets of operating conditions can be identified at first as: robust, semi-robust and best SBR. The RF applied power is the most critical parameter and lower applied power improved the sensitivity as it leads to lower background signals that can be crucial in trace analysis of complex matrices. A preliminary evaluation based on LOD, SBR and $\mathrm{RSD}_{\text {blank }}$ indicates that the semi-robust condition is more suitable for the determination of REEs by ICP OES.

\section{Acknowledgments}

Financial support from Conselho Nacional de Desenvolvimento Científico e Tecnológico (CNPq) and Fundação de Amparo à Pesquisa do Estado de Minas Gerais (FAPEMIG - PPM-00433-10) is gratefully acknowledged. ICP OES facilities were made available at the Laboratório de Geoquímica (LGqA/DEGEO/UFOP) by Dr. Hermínio Arias Nalini Junior.

\section{References}

1. Sapkota,A.; Krachler,M.; Scholz, C.; Cheburkin,A. K.; Shotyk,W.; Anal. Chim. Acta 2005, 540, 247.

2. Navarro, M. S.; Ulbrich, H. H. G. J.; Andrade, S.; Janasi, V. A.; J. Alloys Compd. 2002, 344, 40.

3. Jaron, I.; Kudowska, B.; Bulska, E.; At. Spectrosc. 2000, 21, 105.

4. Trevizan, L. C.; Nóbrega, J. A.; J. Braz. Chem. Soc. 2007, 18, 678.

5. Tatro, M. E.; Amarasiriwardena, D. In Encyclopedia of Analytical Chemistry; Meyers, R. A., ed.; John Wiley \& Sons: Chichester, 2008, p. 12.

6. Khorge, C. R.; Chakraborty, P.; Saran, R.; At. Spectrosc. 2000, $21,220$.

7. Hoenig, M.; Docekalová, H.; Baeten, H.; Analusis 2000, 28, 419. 
8. Cornell, D. H.; Pure Appl. Chem. 1993, 65, 2453.

9. Martins, T. S.; Isolani, P. C.; Quim. Nova 2005, 28, 111.

10. USGS; Rare Earth Elements - Critical Resources for High Technology, U.S. Geological Survey, Fact Sheet 087-02, 2002. http://pubs.usgs.gov/fs/2002/fs087-02/

11. Balaram, V.; TrAC, Trends Anal. Chem. 1996, 15, 475.

12. Verma, S. P.; Santoyo, E.; Velasco-Tapia, F.; Int. Geol. Rev. 2002, 44, 287.

13. Velitchkova, N.; Velichkov, S.; Daskalova, N.; Spectrochim. Acta, Part B 2007, 62, 386.

14. Olesik, J. W.; Williamsen, E. J.; Appl. Spectrosc. 1989, 43, 1223

15. Romero, X.; Poussel, E.; Mermet, J. M.; Spectrochim. Acta, Part B 1997, 52, 495.

16. Mermet, J.M.; J. Anal. At. Spectrom. 1998, 13, 419.

17. Brenner, I. B.; Zischka, M.; Maichin, B.; Knapp, G.; J. Anal. At. Spectrom. 1998, 13, 1257.

18. Chan, G. C. Y.; Hieftje, G. M.; Spectrochim. Acta, Part B 2006, 61, 642

19. Rao, T. P.; Kala, R.; Talanta 2004, 63, 949.

20. Galley, P. J.; Horner, J. A.; Hieftje, G. M.; Spectrochim. Acta, Part B 1995, 50, 87.

21. Grotti, M.; Magi, E.; Frache, R.; J. Anal. At. Spectrom. 2000 , $15,89$.

22. Mermet, J. M.; Anal. Chim. Acta 1991, 250, 85.

23. Chan, G. C. Y.; Hieftje, G. M.; J. Anal. At. Spectrom. 2008, 23, 181.

24. Nölte, J.; ICP Emission Spectrometry - A Practical Guide; Wiley-VCH Verlag: Weinheim, Germany, 2003.

25. Walsh, J. N.; Buckley, F.; Barker, J.; Chem. Geol. 1981, 33, 141.

26. Petry, C. F.; Pozebon, D.; Bentlin, F. R. S.; At. Spectrosc. 2005, 26, 19.

27. Väisänen, A.; Illander, A.; Anal. Chim. Acta 2006, 570, 93.

28. Velitchkova, N.; Pentcheva, E. N.; Daskalova, N.; Spectrochim. Acta, Part B 2004, 59, 871.

29. da Luz Lopes, W.; Santelli, R. E.; Oliveira, E. P.; de Carvalho, M. F. B.; Bezerra, M. A.; Talanta 2009, 79, 1276.

30. Pozebon, D.; Martins, P.; At. Spectrosc. 2002, 23, 111.

31. Huang, B.; Ying, H.; Yang, P.; Wang, X.; Gu, S.; Zhang, Z.; Zhuang, Z.; Sun, Z.; Li, B.; An Atlas of High Resolution Spectra of Rare Earth Elements for ICP-AES; The Royal Society of Chemistry: Cambrigde, UK, 2000.

32. Ralchenko, Y.; Kramida, A. E.; Reader, J.; National Institute of Standards and Technology: Gaithersburg, MD, 2011. http://physics.nist.gov/asd3 acessed in October 2011.
33. Moutte, J.; Analysis of Geological Materials by ICP-AES in Department of Geology, Saint-Etienne School of Mines; http://www.emse.fr/ moutte/enplasma/IcpReportEng.pdf accessed in August 2011.

34. Santos, J. S.; Teixeira, L. S. G.; Araújo, R. G. O.; Fernandes, A. P.; Korn, M. G. A.; Ferreira, S. L. C.; Microchem. J. 2011, 97, 113.

35. Ferreira, S. L. C.; Korn, M. G. A.; Ferreira, H. S.; da Silva, E. G. P.; Araújo, R. G. O.; Souza, A. S.; Macedo, S. M.; Lima, D. C.; de Jesus, R. M.; Amorim, F. A. C.; Bosque-Sendra, J. M.; Appl. Spectrosc. Rev. 2007, 42, 475.

36. Novotny, I.; Farinas, J. C.; Jia-Liang, W.; Poussel, E.; Mermet, J. M.; Spectrochim. Acta, Part B 1996, 51, 1517.

37. Brenner, I. B.; Zander, A.; Spectrochim. Acta, Part B 2000, 55, 1195.

38. Silva, F. V.; Trevizan, L. C.; Silva, C. S.; Nogueira, A. R. A.; Nóbrega, J. A.; Spectrochim. Acta, Part B 2002, 57, 1905.

39. Capelle, R.; Mermet, J. M.; Robin, J.; Appl. Spectrosc. 1982, $36,102$.

40. Trevizan, L. C.; Vieira, E. C.; Nogueira, A. R. A.; Nóbrega, J. A.; Spectrochim. Acta, Part B 2005, 60, 575.

41. van Veen, E. H.; de Loos-Vollebregt, M. T. C.; J. Anal. At. Spectrom. 1999, 14, 831.

42. Romero, X.; Poussel, E.; Mermet, J. M.; Spectrochim. Acta, Part B 1997, 52, 487.

43. Ivaldi, J. C.; Tyson, J.; Spectrochim. Acta, Part B 1995, 50, 1207.

44. Mermet, J. M.; Poussel, E.; Appl. Spectrosc. 1995, 49, 12A.

45. Chausseau, M.; Poussel, E.; Mermet, J.M.; J. Anal. At. Spectrom. 2000, 15, 1293.

46. Jarvis, I.; Jarvis, K. E.; Chem. Geol. 1985, 53, 335

47. Gásquez, J. A.; DeLima, E.; Olsina, R. A.; Martinez, L. D.; de la Guardia, M.; Talanta 2005, 67, 824.

48. Bentlin, F. R. S.; Pozebon, D.; J. Braz. Chem. Soc. 2010, 21, 627.

49. Poussel, E.; Mermet, J.-M.; Spectrochim. Acta, Part B 1996, 51,75 .

50. Thomsen, V.; Roberts, G.; Burguess, K.; Spectroscopy 2000 , 15,33 .

Submitted: October 6, 2011 Published online: March 15, 2012 